\title{
Efecto de post irradiación láser pulsada en función del plasmón en los coloides con nanopartículas de oro
}

Effect of post irradiation pulsed laser in function of the plasmon in the coloids with golden nanoparticles

\author{
${ }^{1}$ Jesús Rivera Esteban ${ }^{\mathrm{a}},{ }^{2}$ Claver Aldama Reyna ${ }^{\mathrm{a}}$
}

\section{RESUMEN}

Se investigó el efecto de la post irradiación con láser pulsado (Post Laser Irradiation; PLI) visible de $532 \mathrm{~nm}$ en coloides conteniendo nanopartículas de oro, los cuales fueron obtenidos mediante la técnica de ablación láser pulsada (PLAL) de $1064 \mathrm{~nm}$ de longitud de onda sobre muestras sólidas de oro inmerso en agua. El propósito de esta investigación fue buscar nuevas técnicas para la obtención de nuevas morfologías de las nanopartículas de oro y la utilidad de la técnica fotoacústica (PA) para la caracterización de los coloides con nanopartículas de oro (AuNPs). El blanco fue ablacionado con láser pulsado de Nd: YAG, (1064 nm, $37.48 \mathrm{~mJ} /$ pulso de $10 \mathrm{~Hz}$, durante 10 minutos), los espectros de absorción presenta un solo máximo de absorción a $521 \mathrm{~nm}$ característica de las nanopartículas esféricas de oro. Se aplicó la técnica PLI a los coloides con pulsos láser de $532 \mathrm{~nm}$ de longitud de onda, $25.8 \mathrm{~mJ}$ de energía durante 6 minutos. El análisis por dispersión dinámica de la luz (Dynamic Light Scattering; DLS) mostro que las AuNPs con PLI fue de $21 \mathrm{~nm}$ de diámetro lo que resulta menor respecto a los coloides sin PLI. De la investigación se concluyó que la técnica PLI focalizada aplicada a los coloides con AuNPs presenta cambio de tamaño.

Palabras clave: post irradiación con láser pulsado, nanopartículas de oro.

\section{ABSTRACT}

The effect of pulsed laser irradiation (Post Laser Irradiation; PLI) visible from $532 \mathrm{~nm}$ in colloids containing gold nanoparticles was investigated, which were obtained using the $1064 \mathrm{~nm}$ wavelength pulsed laser ablation technique (PLAL) on solid samples of gold immersed in water. The purpose of this research was to seek new techniques for obtaining new morphologies of gold nanoparticles and the usefulness of the photoacoustic technique (PA) for the characterization of colloids with gold nanoparticles (AuNPs). The target was ablated with pulsed laser of Nd: YAG, $(1064 \mathrm{~nm}, 37.48 \mathrm{~mJ} / 10 \mathrm{~Hz}$ pulse, for 10 minutes $)$, the absorption spectra exhibited a single maximum absorption at $521 \mathrm{~nm}$ characteristic of spherical gold nanoparticles. The PLI technique was applied to colloids with $532 \mathrm{~nm}$ wavelength laser pulses, $25.8 \mathrm{~mJ}$ of energy for 6 minutes. Dynamic Light Scattering (DLS) analysis showed that AuNPs with PLI were $21 \mathrm{~nm}$ in diameter, which is smaller compared to colloids without PLI. From the investigation it was concluded that the focused PLI technique 
applied to colloids with NPs-Au shows a change in size.

Keywords: pulsed laser post irradiation, gold nanoparticles.

${ }^{1}$ Universidad Nacional Autónoma de Tayacaja

${ }^{2}$ Universidad Nacional de Trujillo.

${ }^{a}$ Licenciado en Física 


\section{INTRODUCCIÓN}

La Nanociencia y la Nanotecnología son dos disciplinas emergentes con un elevado carácter multidisciplinar y con especial relevancia científica y social (FECYT-2009). Esta disciplina abre las puertas a la compresión de nuevos fenómenos y al descubrimiento de nuevas propiedades y que puede ser utilizables a escala macroscópica y microscópica: El objetivo es el control del comportamiento y la estructura de la materia a nivel atómico y molecular. En la literatura se muestran varias definiciones respecto a un nanomaterial o nanopartícula, así, por ejemplo, la National Science and Technology, NNI, por sus siglas en ingles propuso la siguiente definición: material con dimensiones de aproximadamente entre 1 - $100 \mathrm{~nm}(1 \mathrm{~nm}=10-9 \mathrm{~m})$, donde fenómenos únicos permiten nuevas aplicaciones y que fue reportado por Kaiser et al.,(2014).

Los antecedentes en el uso de la nanotecnología fueron con fines artísticos y que tenían un carácter eminentemente empírico y que son el punto de partida actual para el desarrollo de nanomateriales, como ejemplo citamos la famosa copa de Bronce de Lycurgus, que data del siglo IV d. C. y que posee vidrio coloreado cuya propiedad es que dispersa luz verde y transmite luz roja debido a que contiene nanopartículas metálicas de unos 5 -50 nm que son una aleación de plata y oro, (Barber, 1990).
Guerrero, et. al., (2011), reportaron que a pesar de la novedad de estudios recientes, los cambios en las propiedades ópticas del oro, la plata y otros metales cuando se dividen en partículas de muy pequeñas dimensiones, fueron ya estudiadas desde mucho antes de que surgiese la Nanotecnología, o incluso la Ciencia de Superficies y Coloides como tales. Probablemente, el primer estudio científico moderno sobre el tema sea el famoso tratado: "Relaciones Experimentales del Oro (y otros metales) con la Luz", publicado por Michael Faraday en 1857. Dichos fenómenos ópticos eran ya conocidos y habían sido explotados con la finalidad de proporcionar coloración de vidrios, por ejemplo en las vidrieras de iglesias y catedrales.

El método de reducción química es una de las más frecuentes utilizado para la preparación de soluciones coloidales de metales. Consiste en la reducción de una sal del metal mediante un reductor químico. La naturaleza del reductor determina en gran medida la forma, el tamaño y las propiedades eléctricas de las partículas resultantes, por lo tanto, su elección dependerá de la aplicación ulterior de las nanopartículas obtenidas, (Sánchez-Cortez, 2004). Pero los coloides producidos por el método químico están generalmente contaminados con los subproductos residuales tales como iones y agentes reductores. Liang, et al., (2004), describen el método de ablación con láser 
láser para preparar nanopartículas metálicas (MNPs) en soluciones utilizando el láser con parámetros definidos, Kabashin et al., (2003) describen las ventajas de este método incluyen la relativa simplicidad del procedimiento y la ausencia de reactivos químicos en la preparación final. Pero los anchos de las distribuciones de tamaños de las nanopartículas (NPs) preparadas mediante esta técnica tienden a ser grandes debido a la aglomeración, tanto a la post-irradiación de nano clusters y de la eventual expulsión de los relativamente grandes fragmentos del blanco durante el proceso de la ablación con láser. Alanazi, et al., (2010) reportó que, la capacidad del oro para producir calor después de absorber la luz ofrece un uso medicinal llamado terapia fototérmica. Para Fernández, (2014) recientemente, el interés por este material en forma de partículas de tamaño nanométrico se ha visto renovado y fortalecido debido a los comportamientos físicos que exhiben estos sistemas, los cuales presentan nuevas dependencias con el tamaño y forma del material. De este modo, el estudio de este nuevo método resulta fundamental y podría explotarse en el diseño de nuevos dispositivos y tecnologías así como en el desarrollo de nuevas técnicas terapéuticas y diagnósticos, (Daniel, M. y Astruc, D., 2004).

Actualmente, la técnica de ablación láser pulsada en líquidos (PLAL) es ampliamente utilizada para la generación de NPs, debido a que no genera residuos tóxicos y además las superficies de las NPs se encuentran ligadas, lo cual permite la posterior funcionalización con moléculas de interés particular para el investigador. La PLAL, está ganando un creciente interés gracias a una serie de ventajas prometedoras, que incluyen: la sostenibilidad ambiental, de fácil montaje experimental, que se producen completamente libre de contaminantes no deseados o reactivos de síntesis peligrosas y fueron estudiadas por Amendola y Meneghetti (2012), Nikov et al., (2013) y Dell'Aglio et al., (2015), que consiste básicamente en hacer incidir un láser de alta potencia de manera pulsada sobre una superficie de un blanco sólido, sumergido en un medio líquido, estos trabajos se complementan con los desarrollados por, Elsayed et al., (2013) y Hisham et al., (2012), quienes afirman, por ejemplo, que si la energía del pulso láser y el grado de focalización son los adecuados, la densidad de energía depositada sobre el blanco puede ser lo suficiente para elevar la temperatura en varios miles de grados centígrados y evaporar el metal del blanco hasta llevarlo al estado del plasma, dicho plasma se propaga en dirección perpendicular a la superficie del blanco. Para López, (2013), una variación de esta técnica es colocar el blanco dentro de un líquido, de manera que el plasma generado se condense inmediatamente en el líquido en forma de nanopartículas y para Kang, H. W. y Welch, A. J. (2007) 
una característica que debe tener el líquido empleado es la transparencia a la longitud de onda del láser usado, así como la importancia a tener en cuenta es el espesor del medio líquido. Pyatenko et al., (2013) lograron la producción de nanopartículas pequeñas $(2-5 \mathrm{~nm})$ de plata, mientras que Sylvestre, et al., (2004) estudiaron diferentes mecanismos de remoción de material durante la ablación láser de femtosegundo trataron de encontrar las condiciones necesarias para la fabricación de pequeños y casi monodispersas partículas de oro en el agua. Mafuné et al., (2002) reportaron otra forma para el control del tamaño en la preparación de nanopartículas, la combinación del método de ablación láser con la irradiación de las nanopartículas inducida por láser. La irradiación de nanopartículas con láser pulsado, con longitud de onda en el intervalo correspondiente al plasmón de superficie, puede causar la reducción de tamaño. Tarasenko, N. et al., (2006) reportaron los cambios morfológicos inducidos por láser en los coloides de oro y plata que fueron obtenidos por la técnica de PLAL y caracterizados por espectroscopia de absorción óptica y microscopía electrónica. Se encontró que la morfología de las nanopartículas resultantes estaba determinados por los procesos de la fragmentación inducida por láser que ocurre simultáneamente con un crecimiento de partículas que depende de la fluencia, longitud de onda del láser y pos irradiación. La técnica de Post Laser
Irradiation (PLI) (Takeshi Tsuji, et al., 2012), permite obtener NPs con tamaños diferentes a las producidas por la técnica PLAL.

Por otro lado, la resonancia de los plasmones de superficie (PSR), se deben a las oscilaciones colectivas de electrones restringidos en pequeños volúmenes metálicos. Para que este fenómeno ocurra, la partícula tiene que ser mucho menor que la longitud de onda de la luz incidente. Como el resto de los núcleos y sus electrones internos no se han desplazado, constituyen una carga opuesta positiva (red catiónica). Esta separación de cargas actuará como una fuerza restauradora del equilibrio. En partículas pequeñas se produce un dipolo, pero en partículas grandes (a partir de $30 \mathrm{~nm}$ ) se produce un cuadrupolo y en general multipolos, (Cruz D., 2011).

Este trabajo de tesis doctoral está principalmente motivado por nuestro interés en comprender y controlar la respuesta plasmónica de las NPs coloidales del Au; las AuNPs, exhiben propiedades físicas, químicas y biológicas, que son intrínsecas a su tamaño nanométrico, así las aplicaciones es debidos a que presentan propiedades fototérmicas, por la que al ser activadas en presencia de láser pulsado, desprenden calor, (Bellucci, S. Ed., 2008), (Liu, J., \& Jiang, G. Eds., 2015). En general las NPs metálicas presentan múltiples aplicaciones, para tales aplicaciones, las 
NPs deben ser estables, con tamaños pequeños bien definidos y de estrecha distribución, con la posibilidad de funcionalizar la superficie y sobre todo libre de cualquier subproducto, (Salminen, 2013).

El campo de estudio en esta tesis es la síntesis de AuNPs en estado coloidal utilizando la técnica PLAL, aplicación de la técnica PLI, caracterización por espectroscopia fotoacústica pulsada y UV-Vis. La técnica PLAL es un fenómeno muy complejo, por la cantidad de variables que presenta y explorar todos los parámetros a la vez resulta complicado, pero sí es importante tenerlas en cuenta al momento del análisis de los resultados. Para la delimitación del trabajo, consideramos las variables de estudios estas son: la concentración de los coloides con AuNPs.

El tema de investigación es importante debido fundamentalmente, a las potenciales aplicaciones que se pueden explotar en diversos campos, como la medicina, electrónica, medio ambiente. En la literatura se encuentra abundante información respecto a la técnica PLAL, pero aún no han sido explorados completamente los fenómenos físico y químicos de esta técnica. Para llevar a cabo los estudios de los efectos de los parámetros del láser en la síntesis de los coloides y la características de las nanopartículas obtenidas, tales como: el diámetro promedio, la distribución de tamaño, la concentración y la morfología, se utilizaron varias técnicas: fotoacústica pulsada (PA), espectroscopia ultravioleta visible (UV-Vis), espectroscopia de absorción atómica (AAS; Atomic Absorption Spectrometer), y la dispersión dinámica de la luz, (DLS; Dynamic Light Scattering), respectivamente.

Nuestro objetivo general fue estudiar el efecto de la PLI en los coloides con nanopartículas de oro, producidas por la técnica PLAL. Mientras que los objetivos específicos fueron: síntesis, post irradiación y caracterización de coloides de AuNPs utilizando la técnica PLI. Lo novedoso de esta tesis fue el cambio de tamaño en los diámetros promedios de las AuNPs, debido al mecanismo de la post irradiación láser.

La presentación se inicia con una breve introducción general para abordar la nanoestructuras y antecedentes históricos. Se presenta el estado actual de la investigación abordando aspectos generales de las nanopartículas metálicas, También se abordara brevemente el estado del arte, se da a conocer aspectos relevantes de los resultados reportados por otros autores, que sirvieron de conocimiento previo para la elaboración de esta investigación.

\section{MATERIALES Y MÉTODOS}

\section{OBJETO DE ESTUDIO}

Efecto de la irradiación con pulsos láser 
de longitud de onda visible de $532 \mathrm{~nm}$ en los coloides con AuNPs. Los coloides fueron obtenidos mediante la técnica PLAL de $1064 \mathrm{~nm}$ de longitud de onda sobre muestra sólida de oro inmerso en agua ultrapura.

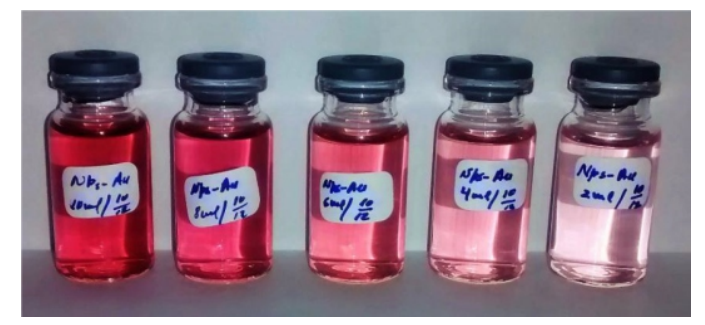

Figura 1. Imagen de los coloides con diferentes concentraciones de AuNPs. El color más intenso (coloide menos transparente) de la dilución corresponde a la de concentración $0.854 \mathrm{mg} / \mathrm{l}$.

Se muestran los coloides con AuNPs, preparadas en agua ultrapura por el método PLAL. Los colores diferentes observados se deben a las diferentes concentraciones, así el de menor concentración $(0.170 \mathrm{mg} / \mathrm{l})$ está ubicado al lado derecho de la Figura 1, mientras que el coloide de mayor concentración $(0.854 \mathrm{mg} / \mathrm{l})$ es la que aparece al lado izquierdo de la Figura 1.

\section{INSTRUMENTACIÓN}

Equipos y materiales del Laboratorio de Óptica y Láseres de la Universidad Nacional de Trujillo:

a. Para la síntesis de los coloides de nanopartículas de oro:
- Láser de Nd: YAG Brilliant de Quantel, con longitud de onda de 1064 $\mathrm{nm}, 10 \mathrm{~ns}$ de duración del pulso y frecuencia de repetición de $10 \mathrm{~Hz}$.

- Lente convergente de $100 \mathrm{~mm}$ de distancia focal.

- Espejo de alta reflectancia para radiación láser pulsada de alta energía IR y visible.

- Blanco de oro de 24 quilates y dimensiones $15 \mathrm{~mm}$ x $16 \mathrm{~mm}$ x 1.05 $\mathrm{mm}$

- Un vaso de precipitado pyrex de 25 $\mathrm{ml}$.

- $20 \mathrm{~mL}$ de agua ultrapura (18 M $\Omega \mathrm{cm})$.

- Lentes de protección para radiación láser infrarroja.

- Dispositivo giratorio sobre el cual se fija el vaso de precipitado. Este mecanismo evita la ablación en una misma zona del blanco.

b. Para la detección de las señales fotoacústicas de los coloides de AuNPs:

- Láser de Nd: YAG Brilliant de Quantel, con longitud de onda $532 \mathrm{~nm}$, 10 ns de duración del pulso, frecuencia de repetición de $10 \mathrm{~Hz}$. (Se trata del mismo láser que se utilizó para la producción de los coloides con 1064 $\mathrm{nm}$, si se acopla el doblador aparece el $532 \mathrm{~nm}$ ).

- Osciloscopio digital Tektronix DPO $3054500 \mathrm{MHz}, 2.5 \mathrm{GS} / \mathrm{s}$ de cuatro canales.

- Computadora personal HP 450 Intel Core i3. 
- Sensor piezoeléctrico de $254 \mathrm{KHz}$ de frecuencia de resonancia (PZT), adherido a la cubeta de cuarzo, para detectar las señales fotoacústicas.

- Fotodiodo de respuesta rápida.

- Lámina de vidrio de 76 × 26 × $1 \mathrm{~mm} 3$, la cual se utilizó para dividir el haz láser en dos componentes. Un componente se dirige al fotodiodo y el otro se dirige a la cubeta de cuarzo que contiene al coloide.

- Lentes de protección para radiación láser verde (532 nm).

\section{MÉTODOS Y TÉCNICAS}

Describiremos los trabajos realizados en el Laboratorio de Óptica y Láseres (LOYL) del Departamento Académico de Física de la Universidad Nacional de Trujillo, y que, corresponde a la producción, post irradiación y caracterización de los coloides con AuNPs. En esta parte se ha utilizado, espectroscopia óptica UV-Vis, fotoacústica pulsada (PA), (AAS) y DLS.

\section{Síntesis de los coloides con AuNPs.}

Se sintetizaron coloides con NPs-Au por el método PLAL. El blanco fue ablacionado con radiación infrarroja de longitud de onda $1064 \mathrm{~nm}$ proveniente de un láser $\mathrm{Nd}$ : YAG, con $12 \mathrm{~mJ}$ de energía a $10 \mathrm{~Hz}$, durante 10 minutos. Al considerar la solución principal, se prepararon cinco muestras en dilución para variar la concentración la cual fue medida por
AAS.

Para direccionar el haz láser sobre la placa se utilizó un espejo y para focalizar el haz se usó una lente, tal como se muestra en la Fig. 2. Además, se empleó una plataforma giratoria sobre la cual se adhirió el vaso conteniendo a la lámina de oro y agua ultrapura, esta cumple dos funciones: evitar la ablación de la placa en una única zona y agitar la solución coloidal, en la Fig. 3 (a) se muestra una fotografía de la placa de oro con las trazas ocasionadas por la acción de los pulsos láser.

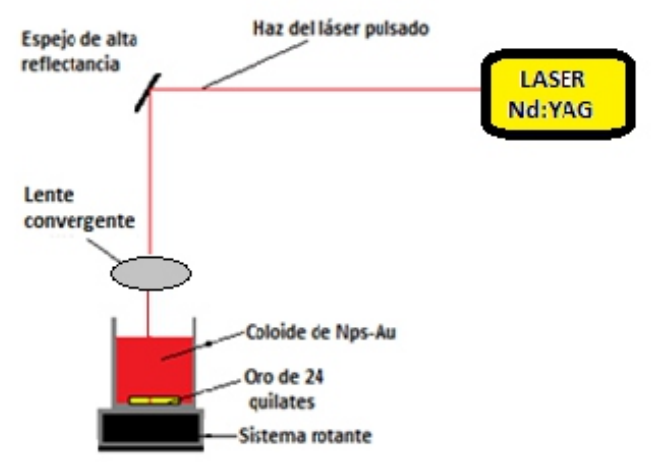

Figura 2. Esquema experimental completo para producir coloides con AuNPs, por la técnica PLAL utilizando un láser pulsado $\mathrm{Nd}$ : YAG.

Se fijaron como parámetros la energía del láser en $37.48 \mathrm{~mJ} /$ pulso y a una frecuencia de repetición de $10 \mathrm{~Hz}$. Bajo estas condiciones se prepararon dos muestras con $20 \mathrm{ml}$ cada una haciendo una solución única de $40 \mathrm{ml}$ coloides con AuNPs. Luego se formaron cinco muestras por disolución. Se aplicó el método PLI utilizando el mismo láser pero con 
longitud de onda de $532 \mathrm{~nm}$, con energía de $0.5 \mathrm{~mJ} /$ pulso, durante 6 minutos, esto consistió básicamente en retirar el blanco de oro y aplicar PLI directamente al coloide.

De las cinco muestras en disolución con concentraciones diferentes se dividieron cada una en dos muestras la primera para ser caracterizada por espectroscopia UVVis y la segunda muestra por espectroscopia fotoacústica pulsada.

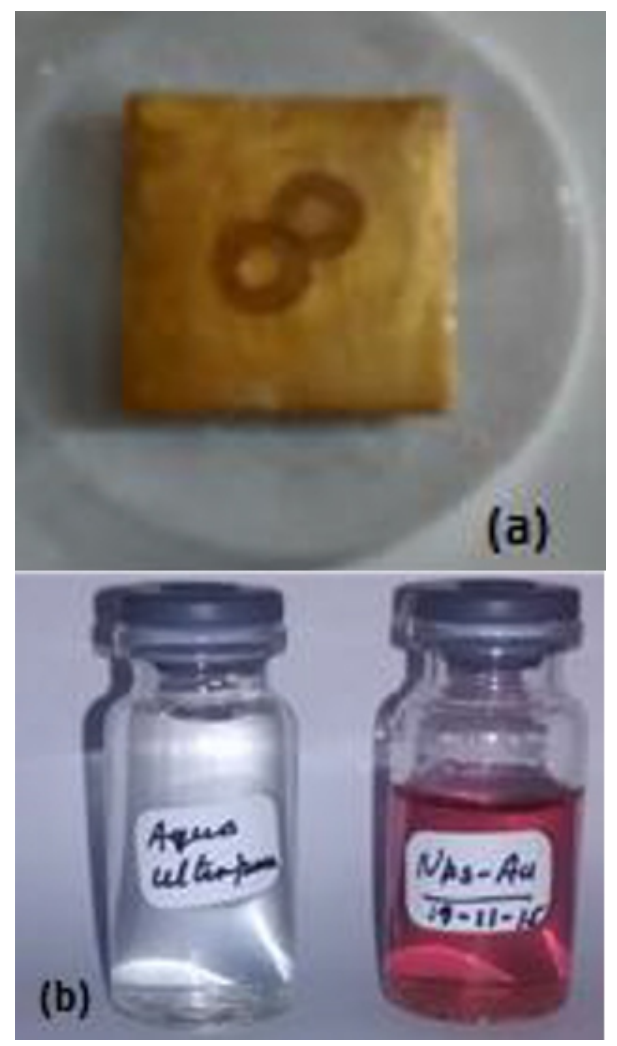

Figura 3. (a) placa de oro de alta pureza utilizada para producir coloides con AuNPs, se observa las zonas irradiadas por el láser durante la ablación. (b) coloide con AuNPs en contraste con agua ultrapura.

En la Figura 3 se presenta una fotografía de la placa de oro mostrando las zonas irradiadas por el haz láser durante la ablación, se muestran también, el medio líquido utilizado (agua ultrapura) y el oro en estado coloidal obtenido.

\section{Detección de las señales PA de los coloides con AuNPs.}

Las señales fotoacústicas de los coloides con NPs-Au fueron obtenidas al considerar la técnica de fotoacústica pulsada. (Figura 4). Se utilizó el segundo armónico de un láser Nd: YAG (532 nm) a una frecuencia de repetición de $10 \mathrm{~Hz}$, en razón de que los coloides metálicos como el oro, la plata absorben la energía en la región visible del espectro electromagnético. Las señales fotoacústicas fueron producidas debido a la interacción de los coloides con la radiación de $532 \mathrm{~nm}$. Las señales fotoacústicas se detectaron mediante un sensor PZT de $254 \mathrm{kHz}$ adherido a una de las caras de una cubeta de cuarzo de dimensiones $1 \mathrm{~cm}$ x $1 \mathrm{~cm}$ x $5 \mathrm{~cm}$, la cual contenía $3 \mathrm{ml}$ del coloide de nanopartículas de oro, estas señales fueron adquiridas con un osciloscopio digital (DPO 3054 de $500 \mathrm{MHz}, 2.5 \mathrm{GS} / \mathrm{s}$ ) y los datos exportados a una computadora para su posterior análisis. Se utilizó un fotodiodo como mecanismo de disparo del osciloscopio para que este pueda registrar las señales detectadas por el sensor PZT.

La energía del haz láser utilizado para la irradiación de los coloides de 
La energía del haz láser utilizado para la irradiación de los coloides de nanopartículas de oro fue de $0.5 \mathrm{~mJ} /$ pulso y está por debajo del umbral de ablación del coloide (régimen termoelástico) donde no se deforman las fuentes de donde proviene la señal PA y que proporcionara información acerca de las Nps sin modificarlas, Alba (2013).

\section{Caracterización por espectroscopia de absorción atómica.}

Las concentraciones de oro presentes en los coloides con AuNPs, fueron caracterizados al utilizar el equipo Perkin Elmer, Atomic Absorption Spectrometer PinAAcle900F, del Laboratorio de Química Instrumental de la Universidad Nacional de Trujillo.

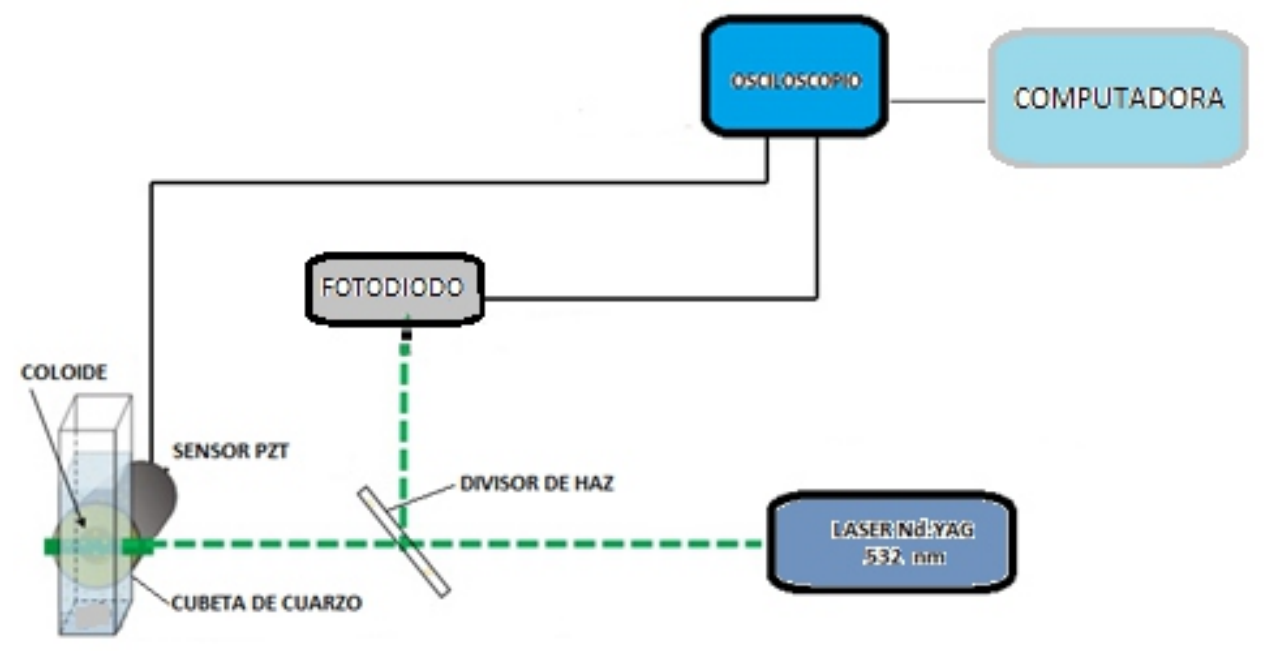

Figura 4. Montaje experimental para la detección de las señales fotoacústicas de los coloides de AuNPs.

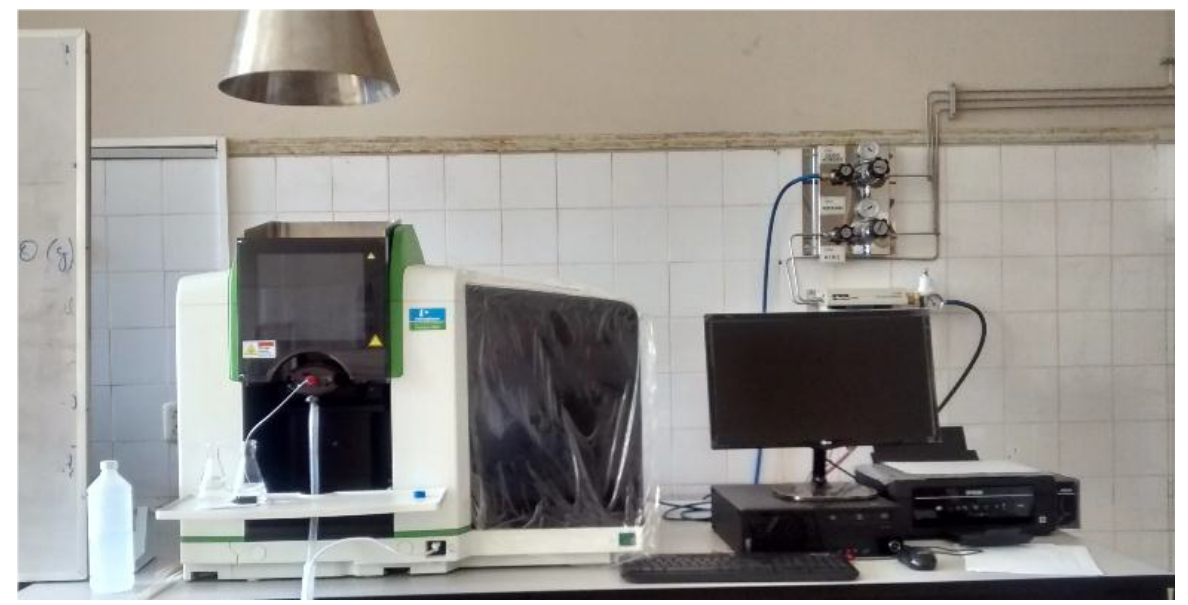

Figura 5. Equipo de absorción atómica del Laboratorio de Química Instrumental, de la Universidad Nacional de Trujillo. 


\section{Caracterización por Espectroscopia} Ultravioleta-Visible (UV-Vis).

Es una técnica espectroscópica basada en las transiciones electrónicas en la región UV (380 nm-780 nm) del espectro electromagnético. En el caso de las AuNPs los espectros de UV-Vis nos sirven para monitorear el desplazamiento del SPR, que está asociado al tamaño y forma de las AuNPs y proveen información de la unión de las moléculas a la superficie de las AuNPs, además de evaluar la estabilidad de las suspensiones coloidales.

\section{Caracterización por Dispersión Dinámica de la luz (DLS).}

La dispersión dinámica de luz (DLS), se utiliza para medir el tamaño de una partícula en el rango sub micrométrico utilizando el movimiento browniano en medios acuosos. En esta técnica, un haz de láser se envía a las nanopartículas suspendidas y la dispersión de luz es detectada por un fotomultiplicador, que se coloca en un ángulo de dispersión de 900. La luz dispersada provoca una señal, que es recibida por el fotomultiplicador. Las partículas pequeñas se difunden más rápidamente $\mathrm{y}$ conducen $\mathrm{a}$ una mayor fluctuación de la intensidad de la luz dispersada en comparación para partículas más grandes (Pecora R. 2000). Los resultados se pueden dar en modo: Intensity Weighting, Volume Weighting, o Number Weighting, es decir ponderando la intensidad de las nanopartículas, el volumen de las nanopartículas o el número de las nanopartículas, respectivamente, (nosotros damos los resultados ponderando el número de las nanopartículas).

\section{RESULTADOS}

De una solución de $40 \mathrm{ml}$ con coloides de AuNPs preparados por el método PLAL se formaron cinco muestras por disolución, estas fueron caracterizadas por UV-Vis (Fig.4) posteriormente fueron aplicadas el método PLI.

\section{Síntesis de coloides con NPs-Au, por el método de PLAL.}

Se colectaron los espectros de absorción de cada muestra, para cada espectro, se observó, un único máximo (pico) en el intervalo visible del espectro electromagnético, lo cual es característico de AuNPs esféricas. Al comparar diferentes espectros no se evidencio grandes corrimientos de la posición del máximo relacionado con el plasmón de superficie.

Al realizar los análisis de los resultados obtenidos por la técnica de DLS, se confirmó la presencia de NPs esféricas con diámetros de aproximadamente 21 $\mathrm{nm}$.

Caracterización por espectroscopia UV-Vis, de los coloides con AuNPs 
producidas por la técnica PLAL.

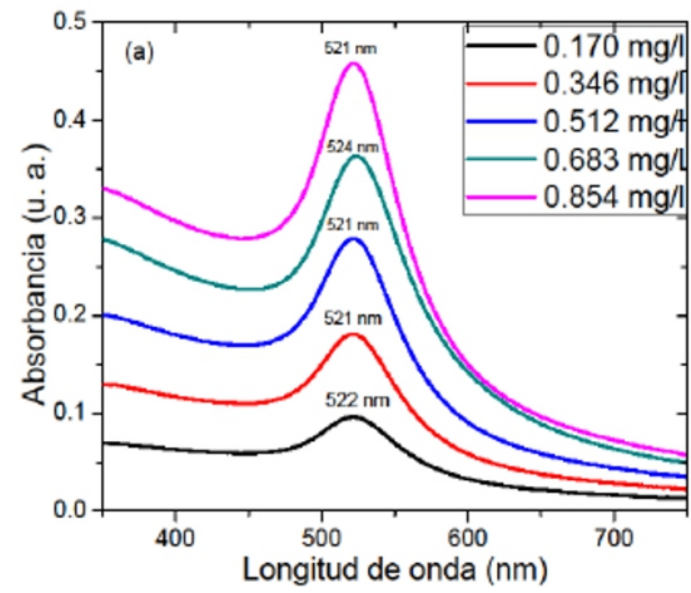

Figura. 6. Espectro de absorción de los coloides con AuNPs, obtenida por espectroscopia UV-Vis, se refiere a las muestras diluidas y que fueron obtenidas por la técnica PLAL.

Los espectros de absorción se muestran en la Figura 6, muestran los espectros de absorción de los coloides con AuNPs para diferentes concentraciones. Se puede observar un único máximo de absorción en la región visible del espectro electromagnético, relacionado con la resonancia del plasmón de superficie (PSR); este resultado sugiere que las AuNPs presentes en los coloides presentan forma esférica y tienen tamaño de algunas decenas de nanómetros, la forma del espectro permite inferir que la forma y tamaño de las nanopartículas en promedio permanecen sin cambio apreciables, tal como lo describen Jain et al., (2006).

La Figura 7, muestra los espectros de absorción de los coloides con AuNPs, se utilizó el láser pulsado $\mathrm{Nd}$ : YAG de 532 $\mathrm{nm}$ de longitud de onda durante 6 minutos y con una energía de irradiación de 25.8 $\mathrm{mJ} /$ pulso. En todos los casos se observa que el espectro de absorción presenta menor intensidad con respecto al espectro de absorción de la Fig. 6.

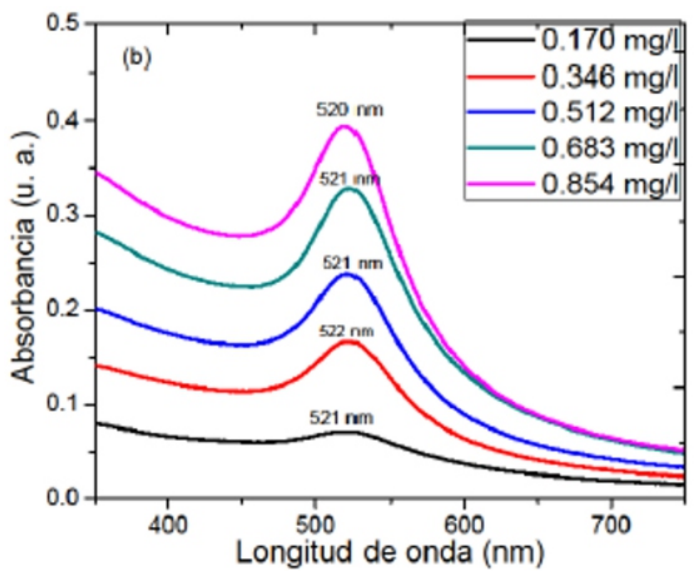

Figura 7. Espectro de absorción de los coloides con AuNPs diluidas, producidas por el método PLAL y que fueron tratadas por el método PLI.

La técnica UV-Vis nos permite evidenciar el plasmón superficial de las AuNPs, el cual refleja la oscilación colectiva de los electrones de conducción de las nanopartículas, debido a las interacciones de los mismos con la luz incidente. La interacción ocasiona una polarización temporal y por ello la formación de un dipolo oscilante. El dipolo oscila a la frecuencia de la luz incidente. Existe una frecuencia de resonancia para la cual la interacción entre la nanopartícula y la luz origina un máximo de absorción. Este máximo depende de la forma y tamaño de la nanopartícula y el medio circundante. 
Como consecuencia de la fuerte interacción entre los electrones y la luz incidente, se produce una oscilación coherente de los electrones confinados en la superficie de las nanopartículas metálicas, en la interfaz metal-dieléctrico; la excitación electromagnética se llama polaritón del plasmón de superficie; el polaritón es una cuasi-partícula híbrida resultante del fuerte acoplamiento entre la luz y los electrones libres de una nanopartícula. Para determinadas frecuencias de la energía incidente, se produce un acoplamiento entre las frecuencias de la onda incidente y de la oscilación del polaritón; este fenómeno, como vimos anteriormente es la SPR (Y. Wang, 2011), (J. J. Mock, et al., 2002). Al comparar la figura 4 con la figura 5 , de los espectros de absorción de los coloides con AuNPs con diferentes concentraciones, se puede observar que cada muestra post irradiada presenta una disminución en la intensidad del su máximo de absorción en comparación con su similar en valor de concentración. Los datos que se presentan en la tabla $\mathrm{N}^{\mathrm{o}} 1$, provienen de la figura $6, \mathrm{y}$ la figura 7 , en la primera columna se presenta las concentraciones en $\mathrm{mg} / \mathrm{l}$.

Tabla 1. Parámetros principales de los espectros de absorción de las AuNPs. El máximo de absorción del espectro relacionado con la SPR, densidad óptica (DO) ancho de banda (FWHM, bandwidth). Como se sabe estos parámetros están relacionadas con la concentración, tamaño y forma de las nanopartículas.

\begin{tabular}{ccccccc}
\hline $\begin{array}{c}\text { CONCENTRACIÓN } \\
(\mathbf{m g} / \mathbf{L})\end{array}$ & $\begin{array}{c}\text { PICO MÁXIMO (nm) } \\
\text { PLAL }\end{array}$ & PLI & \multicolumn{2}{c}{$\begin{array}{c}\text { DENSIDAD ÓPTIMA } \\
\text { PLAL }\end{array}$} & PLI & \multicolumn{2}{c}{ FWHM(nm) } \\
PLAL & PLI \\
\hline 0.17 & 522 & 521 & 0.0346 & 0.0105 & 44 & 37 \\
0.346 & 521 & 522 & 0.0683 & 0.0509 & 45 & 44 \\
0.512 & 521 & 521 & 0.1067 & 0.0729 & 45 & 43 \\
0.683 & 524 & 521 & 0.135 & 0.103 & 47 & 46 \\
0.854 & 521 & 520 & 0.1725 & 0.1158 & 44 & 44 \\
\hline
\end{tabular}

En la figura 8, se presenta la relación de la absorbancia máxima en función de la concentración, obteniéndose una relación lineal, lo cual nos indica que a mayor concentración de AuNPs en el coloide se obtiene mayor absorbancia.

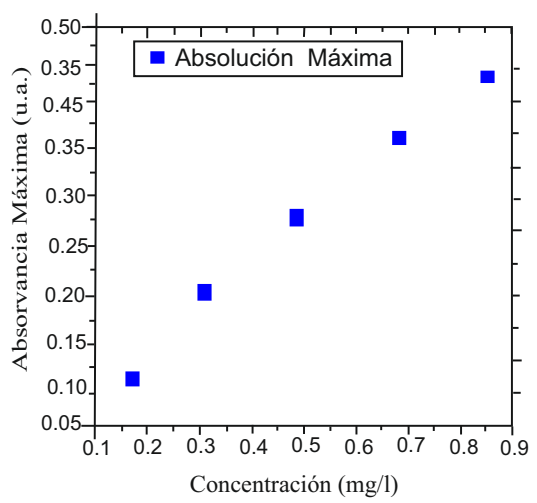

Figura 8. Absorbancia máxima de los coloides con AuNPs con PLI, en función de su concentración, con tendencia es lineal, con un coeficiente de Pearson de 0.99641. 


\section{Caracterización por fotoacústica de las} AuNPs.

En la Figura 9, se muestra una típica señal fotoacústica de un coloide con AuNPs tal como fue obtenida con el osciloscopio. Se visualiza como una curva de voltaje vs tiempo. En la literatura se reporta únicamente el análisis de las variaciones del primer pico de la señal fotoacústica, sin embargo se pueden realizar otros tipos de análisis, por ejemplo la variación de la señal pico-pico entre el primer máximo y el primer mínimo, o bien una cantidad que es el promedio temporal de toda la señal registrada, análogo al análisis RMS. (Raíz cuadrática media), como lo explican, Castañeda, et al., (1999). Los resultados presentados aquí se refieren a la variación del primer pico de la señal. Alba, (2013) reporta que es importante reconocer las diferentes partes que la conforman las señales adquiridas, por lo que bastará con reconocer dos secciones; la primera se debe a la luz esparcida por la muestra y es captada por el PZT, el cual absorbe la luz directamente y produce una señal PA, referido a la posición de la señal de disparo (trigger). La amplitud de esta parte de la señal es una medida indirecta del esparcimiento que tiene la muestra, es decir, entre más luz esparza la muestra, mayor será esta parte de la señal PA. La segunda parte de la señal a considerar se deberá directamente a las fuentes PA suspendidas en la muestra, los rebotes de las ondas acústicas que ocurran dentro de la celda. Se debe señalar que la celda PA, como tal, también produce una señal, pero es muy pequeña comparada con las otras señales estudiadas.

La evolución temporal de las señales PA, que se muestran en la figura 9, fueron detectadas por el sensor PZT de respuesta rápida de $254 \mathrm{kHz}$ y corresponden a la interacción de los pulsos láser con energía de $0.5 \mathrm{~mJ} /$ pulso con las AuNPs coloidal. Se puede observar un comportamiento diferente de las señales y que estas aumentan en amplitud gradualmente en relación con las diferentes concentraciones. Así puede decirse que la técnica PA es capaz de detectar los cambios mínimos en la variación de la concentración de las AuNPs en el coloide. Para mejorar la relación señal a ruido como menciona Barreiro, et al., (2008), debido a ruido exterior, acústico y eléctrico, se promedia la señal, nosotros promediamos sobre 16 pulsos del láser. Como dijimos anteriormente, el primer pico corresponde a la señal acústica longitudinal y los picos subsecuentes se deben a los rebotes de la señal y del PZT, se observa que las amplitudes de la señal aumentan conforme aumenta la concentración de las AuNPs en el coloide, una excepción es para la muestra 0.854 $\mathrm{mg} / \mathrm{L}$, cuya amplitud decae, esto es probablemente debido a las fluctuaciones de la energía del láser, aunque podemos decir que no tenemos una evidencia al respecto.

El análisis de la señal PA generada por 
NPs metálicas es complejo debido a la dependencia de las propiedades físicas, tanto de la NPs y los alrededores del ambiente.

En la figura. 10, muestra al máximo de absorción en función de la concentración, cuya relación es ascendente conforme se aumenta la concentración. Por lo tanto, se comprueba que a mayor concentración de AuNPs la señal PA se incrementa.

El objetivo fundamental de la presente tesis consistió en obtener la respuesta PA de los coloides con Nps-Au; obtenidos por la técnica PLAL. Como se indicó nosotros obtuvimos las NPs-Au en agua

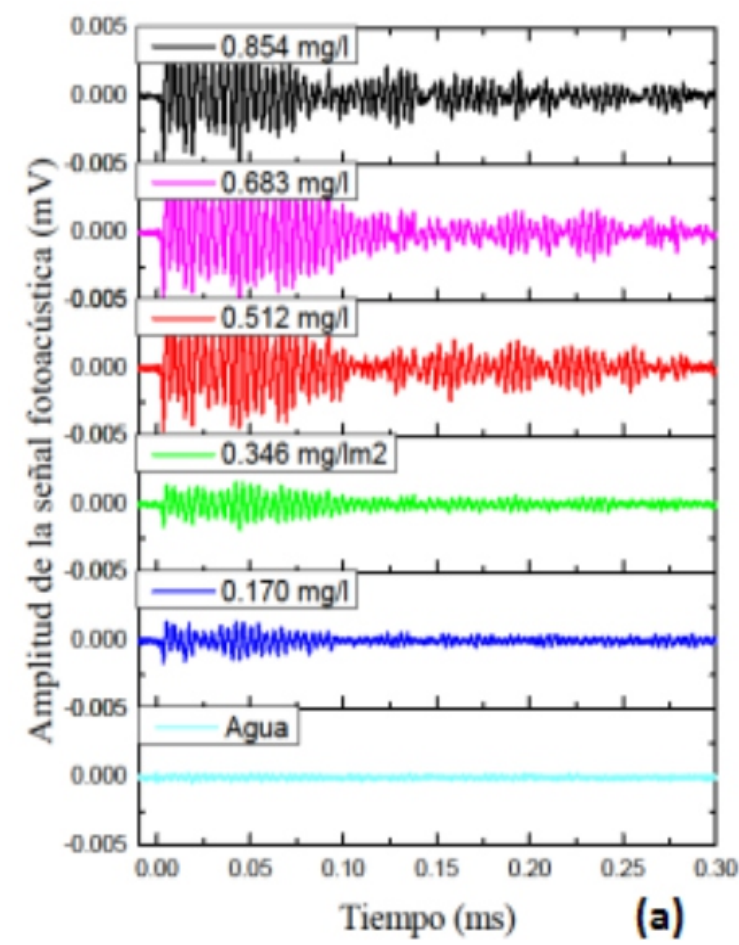

Figura 9. Muestras irradiadas: (a) Respuesta fotoacústica de los coloides con AuNPs en función del tiempo para diferentes concentraciones con PLI ( $=532 \mathrm{~nm}, 0.5 \mathrm{~mJ} /$ pulso de energía) El sistema experimental se muestra en la figura. 3.10. (b) Las mismas señales fotoacústicas se visualiza a una escala más reducida en el tiempo. 


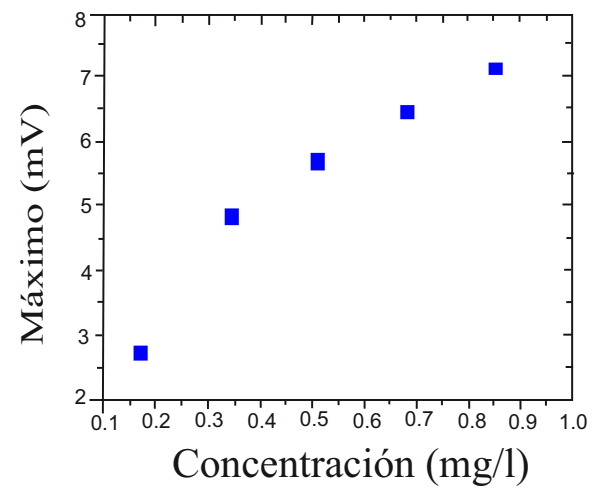

Figura 10. Amplitud máxima de la señal PA en función de la concentración de muestras con PLI.

\section{Obtención de la Concentración por} Absorción Atómica.

La concentración de AuNPs en los coloides se obtuvo al utilizar la técnica de Espectroscopia de Absorción Atómica (AAS). Las concentraciones de oro fueron: $0.170 \mathrm{mg} / 1,0.346 \mathrm{mg} / 1,0.512$ $\mathrm{mg} / 1,0.683 \mathrm{mg} / \mathrm{ly} 0.854 \mathrm{mg} / \mathrm{l}$.

\section{Caracterización por DLS}

La dispersión dinámica de la luz, o Dynamic Light Scattering (DLS, por sus siglas en inglés). Esta técnica es uno de los métodos para determinar el tamaño de partículas. Cuando un haz de luz monocromática, tal como un láser, incide sobre una disolución con partículas esféricas en el movimiento browniano provoca un desplazamiento Doppler cuando la luz llega a la partícula en movimiento, (Zimbone M. et al., 2012).

Las partículas en una solución coloidal siguen el movimiento browniano, como resultado de las continuas colisiones de partículas y el movimiento. Basado en el movimiento browniano, las partículas más pequeñas se moverán más rápido. Un láser que se muestra a través del coloide se dispersará. La intensidad de la luz dispersada depende del movimiento de las partículas coloidales, y el tamaño de partícula así puede determinarse utilizando la relación de Stokes-Einstein. Este método tiene varias ventajas: en primer lugar, la duración de la prueba es corta y es casi todos automatizados de manera que las mediciones de rutina no son extensas. Además no se requiere preparar la muestra, solo se requiere poca cantidad de coloide (ml). A continuación reportamos los resultados:

a. Resultado por espectroscopia DLS de los coloides. Las AuNPs, presentan un diámetro promedio de $22.6 \mathrm{~nm}$,

b. Resultado por espectroscopia DLS de los coloides irradiados. Las AuNPs, presentan un diámetro promedio de 21.0 nm.

\section{DISCUSIÓN}

El objetivo fundamental del presente trabajo consistió en el efecto del PLI en los coloides con AuNPs. Como se indicó nosotros obtuvimos las AuNPs en agua. Los espectros de absorción de las AuNPs para diferentes concentraciones (figura 6), presentan un solo máximo vista a la longitud de onda de $521 \mathrm{~nm}$ y como se sabe está asociada con la resonancia superficial de plasmones (PSR). 
Como sabemos, la concentración está relacionada con la intensidad del espectro de absorción (máximo absorción). El ancho de banda con la homogeneidad de los coloides con AuNPs y la posición del máximo de absorción (pico), está relacionada con la morfología de las AuNPs, si comparamos las Fig. 6 con la figura 7, a través de la tabla No 1 , observamos que existe un corrimiento del máximo de absorción hacia menores longitudes de onda para la muestra la primera muestra (1 nm), cuarta muestra (3 $\mathrm{nm})$ y la quinta muestra $(1 \mathrm{~nm})$, la densidad óptica disminuye para los coloides con PLI para bajas concentraciones y el ancho de banda de los coloides con PLI ha disminuido por lo que podemos inferir que estas partículas son más homogéneos y además más pequeños. Después de la post irradiación de los coloides con AuNPs, puede ocurrir un cambio de forma y reducción de tamaño de las AuNPs. Uno de los mecanismos que pueden ocurrir como consecuencia de la post irradiación de las soluciones coloidales es la fusión y vaporización de las AuNPs, las cuales se deben a las altas temperaturas en el interior de las nanopartículas. La alta temperatura es causada por la gran absorción de la energía del láser y la baja transferencia de calor al agua circundante. (Akinori Takami, et al., 1999). La técnica PLI es útil para preparar coloides con tamaño deseado en soluciones, la fragmentación de estas partículas grandes por una post irradiación adicional de los coloides resultantes es cruciales para la optimización de la distribución de tamaño de partícula en el coloide, Mafuné et al., (2000). En realidad el espectro de absorción es una señal de un colectivo, pues en el coloide hay NPs de variados tamaños, cada una de las NPs contribuye al espectro de absorción, así que, es difícil decir como la altura se ve afectada al tener tantos contribuyentes (NPs de diferentes tamaños).

\section{CONCLUSIONES}

Se obtuvo nanopartículas de oro utilizando la técnica PLAL y se utilizó la técnica PLI para estudiar los coloides con AuNPs mediante espectroscopia óptica UV-Vis., y fotoacústica pulsada, se cuantifico la concentración mediante AAS, por lo que se concluye que la amplitud fotoacústica de los coloides con nanopartículas de oro cambian linealmente con la concentración, así mismo, los coloides con nanopartículas de oro después del PLI focalizada presentan un cambio en tamaño, corroborado por el DLS y que la evidencia de un solo máximo está relacionada con la resonancia de los plasmones de superficie (PSR) mostrando nanopartículas con simetría esféricas.

\section{REFERENCIAS BIBLIOGRAFICAS}

Akinori Takami, Hideaki Kurita, and Seiichiro Koda. (1999). LaserInduced Size Reduction of Noble 
of Noble Metal Particles. Department of Chemical System Engineering, School of Engineering, The UniVersity of Tokyo, Hongo 7-3-1, Bunkyo-ku, Tokyo, 113-8656, Japan.

Alanazi, F. K., Radwan, A. A., \& Alsarra, I. A. (2010). Biopharmaceutical applications of nanogold. Saudi Pharmaceutical Journal, 18(4), 179193.

Alba Rosales, J. E. (2013). Estudio de la Ablación Láser Mediante Fotoacústica Pulsada: Síntesis de Nanopartículas. Tesis Doctoral. Universidad de Guanajuato. México.

Amendola, V., \& Meneghetti, M. (2009). Laser ablation synthesis in solution and size manipulation of noble metal nanoparticles. Physical chemistry chemical physics, 11(20), 3805-3821.

Amendola, V., \& Meneghetti, M. (2012). What controls the composition and the structure of nanomaterials generated by laser ablation in liquid solution?.Physical Chemistry Chemical Physics, 15(9), 30273046.

Bellucci, S. (Ed.). (2008). Nanoparticles and Nanodevices in Biological Applications: The INFN Lectures (Vol. 4). Springer Science \& Business Media.

Barber, D. J., \& Freestone, I. C. (1990). An investigation of the origin of the colour of the Lycurgus Cup by analytical transmission electron microscopy.Archaeometry, 32(1), $33-45$.

Barreiro, N. L., Vallespi, A., Slezak, V. B., Peuriot, A. L., González, M. G., \& Santiago, G. D. (2008, Noviembre). Caracterización de 1 a Espectroscopia Fotoacústica Pulsada del NO2 en aire. In ANALES AFA(Vol. 20, No. 1).

Castañeda Guzmán, R., Villagrán Muñiz, M., \& Saniger Blesa, J. M. (1999). Detención fotoacústica de transiciones de fase en cerámicas ferroeléctricas. Boletín de la Sociedad Española de Cerámica y Vidrio, 38(5), 439-442.

Cruz, D. A., \& Rodríguez, M. C. (2012). Nanopartículas metálicas y plasmones de superficie: Una relación profunda. Avances en Ciencias e Ingeniería, 3(2), 67-78.

Daniel L, M. C., \& Astruc, D. (2004). Gold nanoparticles: assembly, supramolecular chemistry, quantum-size-related properties, and applications toward biology, catalysis, and nanotechnology. Chemical reviews, 104(1), 293 346.

Dell’Aglio, M., Gaudiuso, R., De Pascale, O., \& De Giacomo, A. (2015). Mechanisms and processes of pulsed laser ablation in liquids during nanoparticle production. Applied Surface Science.

Elsayed, K. A., H. Iman, M. A. Ahmed, and R. Ramadam. (2013). Effect of 
focusing conditions and laser parameters on the fabrication of gold nanoparticles via laser ablation in liquid, Optics and Laser Technology, vol. 45, issue 1, pp. 495- 502.

Fernández Cabana T. (2014).

Caracterización de nanopartículas magnéticas y de oro para posibles aplicaciones biomédicas en diagnóstico y terapia. Tesis doctoral. Programa de doctorado en Ingeniería Biomédica. Universidad Politécnica de Madrid.

Guerrero Martínez, A., Álvarez Puebla, R. A., \& Liz Marzán, L. M. (2011, July). Nanoplasmónica basada en Química Coloidal. In Anales de la Real Sociedad Española de Química(Vol. 107, No.3, pp. 221228).

Hishan Iman, Khaled, Mohamed A. Ahmed, Rania Ramdam. (2012). Effect of Experimental Parameters on the Fabrication of Gold Nanoparticles via Laser Ablation. Optics and Photonics Journal, 273-84. Giza Egypt.

J. J. Mock, M. Barbic, D. R. Smith, D. A. Schultz, and S. Schultz, 2002. Forma efectos en resonancia de plasmón de individuo nanopartículas de plata coloidales Jacak et al., (1998)

Jain, P. K., Lee, K. S., El-Sayed, I. H., \& E1-Sayed, M. A. (2006). Calculated absorption and scattering properties of gold nanoparticles of different size, shape, and composition: applications in biological imaging and biomedicine. The Journal of Physical Chemistry B, 110(14), 7238-7248.

Kabashin, A. V., \& Meunier, M. (2003). Synthesis of colloidal nanoparticles during femtosecond laser ablation of gold in water. Journal of Applied Physics, 94(12), 7941-7943.

Kaiser, D. L., Standridge, S., Friedersdorf, L., Geraci, C. L., Kronz, F., Meador, M. A., \& Stepp, D. M. (2014). 2014 National Nanotechnology Initiative Strategic Plan.

Kang, H. W., \& Welch, A. J. (2007). Effect of liquid thickness on laser ablation efficiency. Journal of applied physics, 101(8).

Liang, C., Bruell, C. J., Marley, M. C., \& Sperry, K. L. (2004). Persulfate oxidation for in situ remediation of TCE. II. Activated by chelated ferrous ion.Chemosphere, 55(9), 1225-1233.

Liu, J., \& Jiang, G. (Eds.). (2015). Silver Nanoparticles in the Environment. Springer.

López, G., Morales, R., Olea, O., Sánchez, V., Trujillo, J., Valera, V., Vilches, A. 2013. Nanoestructuras metálicas; síntesis, caracterización y aplicaciones. Libro de Editorial Reverte, Universidad Autónoma del 
Estado de México. 1-50.

Mafuné, F., Kohno, J. Y., Takeda, Y., Kondow, T., \& Sawabe, H. (2000). Formation and size control of silver nanoparticles by laser ablation in aqueous solution. The Journal of Physical Chemistry B, 104(39), 9111-9117.

Mafuné, F., Kohno, J. Y., Takeda, Y., Kondow, T., \& Sawabe, H. (2002). Formation of gold nanoparticles by laser ablation in aqueous solution of surfactant. The Journal of Physical Chemistry B, 105(22), 5114-5120.

Nikov, R. G., Nikolov, A. S., Nedyalkov, N. N., Atanasov, P. A., Alexandrov, M. T., \& Karashanova, D. B. (2013). Processing condition influence on the characteristics of gold nanoparticles produced by pulsed laser ablation in liquids. Applied Surface Science, 274, 105109.

Pecora, R. (2000). Dynamic light scattering measurement of nanometer particles in liquids. Journal of nanoparticle research, 2(2), 123-131.

Pyatenko, A., Wang, H., Koshizaki, N., \& Tsuji, T. (2013). Mechanism of pulse laser interaction with colloidal nanoparticles. Laser \& Photonics Reviews, 7(4), 596-604.

Salminen, T. (2013). Production of Nanomaterials by pulsed laser ablation. Tampereen teknillinen yliopisto. Julkaisu-Tampere University of Technology.
Publication; 1121.

Sánchez-Cortés, S., García, J. V., \& Morcillo, G. (1994). Morphological study of metal colloids employed as substrate in the SERS spectroscopy. Journal of colloid and interface science, 167(2), 428-436.

Sylvestre, J. P., Kabashin, A. V., Sacher, E., Meunier, M., \& Luong, J. H. (2004). Stabilization and size control of gold nanoparticles during laser ablation in aqueous cyclodextrins. Journal of the American Chemical Society, 126(23), 7176-7177. (2004). Stabilization and size control of gold nanoparticles during laser ablation in aqueous cyclodextrins. Journal of the American Chemical Society, 126(23), 7176-7177.

Takeshi Tsuji, Masahata Nakanishi, Takeshi Mizuki, Shogo Ozono, Masaharu Tsuji, and Yasuyuki Tsuboi. Preparation and ShapeModification of Silver Colloids by laser Ablation in Liquids: A Brief Review. Science of Advanced Materials. Vol 4, pp391-400, 2012.

Tarasenko, N. V., Butsen, A. V., Nevar, E. A., \& Savastenko, N. A. (2006). Synthesis of nanosized particles during laser ablation of gold in water. Applied surface science, 252(13), 4439-4444.

Valverde-Alva, M. A., García-Fernández, T., Villagrán-Muñiz, M., SánchezAké, C., Castañeda-Guzmán, R., Esparza-Alegría, E. Márquez- 
Herrera, C. M. (2015). Synthesis of silver nanoparticles by laser ablation in ethanol: A pulsed photoacoustic study. Applied Surface Science, 355, 341-349.

Wang, Y., Plummer, E. W., \& Kempa, K. (2011). Foundations of plasmonics. Advances in Physics, 60(5), 799898.

Zimbone, M., Musumeci, P., Baeri, P., Messina, E., Boninelli, S., Compagnini, G., \& Calcagno, L. (2012). Rotational dynamics of gold nanoparticle chains in water solution. Journal of Nanoparticle Research, 14(12), 1-11.

\section{CCORRESPONDENCIA}

Dr. Jesús Rivera Esteban jgiancolli.61@gmail.com 